\title{
Risk factors for bloodstream infection in patients at a Brazilian hemodialysis center: a case-control study
}

\author{
Dayana Fram*, Meiry Fernanda Pinto Okuno ${ }^{1}$, Mônica Taminato ${ }^{1,2}$, Vinicius Ponzio ${ }^{3}$, Silvia Regina Manfredi4, \\ Cibele Grothe ${ }^{1}$, Angélica Belasco ${ }^{1}$, Ricardo Sesso ${ }^{5}$ and Dulce Barbosa ${ }^{1}$
}

\begin{abstract}
Background: Infection is the leading cause of morbidity and the second leading cause of mortality in patients on renal replacement therapy. The rates of bloodstream infection in hemodialysis patients vary according to the type of venous access used. Gram-positive bacteria are most frequently isolated in blood cultures of hemodialysis patients. This study evaluated risk factors for the development of bloodstream infections in patients undergoing hemodialysis.

Methods: Risk factors associated with bloodstream infections in patients on hemodialysis were investigated using a case-control study conducted between January 2010 and June 2013. Chronic renal disease patients on hemodialysis who presented with positive blood cultures during the study were considered as cases. Controls were hemodialysis patients from the same institution who did not present with positive blood cultures during the study period. Data were collected from medical records. Logistic regression was used for statistical analysis.

Results: There were 162 patients included in the study (81 cases and 81 controls). Gram-positive bacteria were isolated with the highest frequency (72\%). In initial logistic regression analysis, variables were hypertension, peritoneal dialysis with previous treatment, type and time of current venous access, type of previous venous access, previous use of antimicrobials, and previous hospitalization related to bloodstream infections. Multiple regression analysis showed that the patients who had a central venous catheter had an 11.2-fold (CI 95\%: 5.17-24.29) increased chance of developing bloodstream infections compared with patients who had an arteriovenous fistula for vascular access. Previous hospitalization increased the chance of developing bloodstream infections 6.6-fold (Cl 95\%: 1.9-23.09).
\end{abstract}

Conclusions: Infection prevention measures for bloodstream infections related to central venous catheter use should be intensified, as well as judicious use of this route for vascular access for hemodialysis. Reducing exposure to the hospital environment through admission could contribute to a reduction in bloodstream infections in this population.

Keywords: Dialysis, Bacteremia, Risk factors, Staphylococcus aureus

\section{Background}

Infection is the leading cause of morbidity and the second leading cause of mortality among patients on renal replacement therapy [1]. Mortality from all causes in patients on dialysis treatment is $6.5-7.9$ times higher than that of the general population. In patients $\geq 65$ years of

\footnotetext{
* Correspondence: dayana.fram@unifesp.br

'School of Nursing, Paulista School of Nursing, Universidade Federal de São Paulo (Federal University of São Paulo - EPE/UNIFESP), R. Napoleão de Barros 754, São Paulo 04024-002, Brazil

Full list of author information is available at the end of the article
}

age, mortality from infection is two times higher than that in a population of the same age with a diagnosis of diabetes mellitus, neoplasms or congestive heart failure [1]. According to the US Renal Data System [1], the mortality rate at the end of the first year of hemodialysis decreased by $38 \%$ for cardiovascular causes and by $50 \%$ for infectious causes between 2000 and 2010. Among patients on hemodialysis, the mortality rate decreased by 26\% between 1985 and 2000 [1]. When comparing mortality rates between the second and 12th month after initiation of hemodialysis, there was a significant reduction

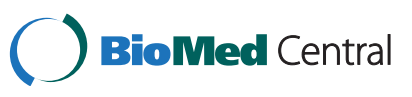

(c) 2015 Fram et al.; licensee BioMed Central. This is an Open Access article distributed under the terms of the Creative Commons Attribution License (http://creativecommons.org/licenses/by/4.0), which permits unrestricted use, distribution, and reproduction in any medium, provided the original work is properly credited. The Creative Commons Public Domain Dedication waiver (http://creativecommons.org/publicdomain/zero/1.0/) applies to the data made available in this article, unless otherwise stated. 
in mortality from all causes, which dropped from 440 to 201 deaths per 1000 patients/year. The mortality rate from infectious causes decreased from 43 to 19.4 deaths per 1000 patients/year [1].

North American data indicate that bloodstream infection (BSI) rates in patients on hemodialysis vary between 0.5 and 27.1 per 100 patients/month depending on the type of venous access used [2]. More recent studies report a reduction in the incidence of BSI in this population between $1.09-0.89$ and $2.04-0.75$ per 100 patients/ month after implementation of specific control measures $[3,4]$. This decrease was attributed to national initiatives for the reduction of healthcare-related infections [5].

The literature reports that the use of a central venous catheter (CVC), hypoalbuminemia, diabetes mellitus, anemia, female gender and colonization by methicillinresistant Staphylococcus aureus (MRSA) are risk factors for the development of BSI in hemodialysis patients [6-10]. Gram-positive bacteria are the most frequently isolated agents in blood cultures from hemodialysis patients, mainly S. aureus $[7,10,11]$.

According to the US Renal Data System, the principal morbidity event for hemodialysis patients is hospitalization for infections, which showed an increase of $43 \%$ between 1993 and 2011. On the other hand, hospitalizations for complications from venous access, showed a decrease of $57 \%[1]$.

Fram et al. reported that the risk factors for morbidity and mortality among patients with BSI on hemodialysis were age, isolation of $S$. aureus, and isolation of a resistant microorganism [12].

Despite the best efforts to prevent BSI specific to hemodialysis patients, BSI still has a large impact [13-16]. Therefore, the objective of this study was to evaluate risk factors for the development of BSI in patients undergoing a Brazilian hemodialysis center.

\section{Methods}

\section{Ethical considerations}

This study was conducted with the approval of the Committee on Ethics and Research at the Federal University of São Paulo.

\section{Design, period and location of the study}

This was a retrospective, case-control study conducted between January 2010 and June 2013 at the hemodialysis satellite unit at the Kidney and Hypertension Hospital of Foundation Oswaldo Ramos (HRIM/FOR) in the city of São Paulo, Brazil. The HRIM/FOR is a nephrology service, considered to be a national and international reference for teaching, research and care activities. It serves an average of 400 patients in the dialysis program/month (221 in hemodialysis). This institution follows rigorous standards for medical record keeping.
Patients with chronic renal insufficiency in a hemodialysis program, $\geq 18$ years of age, with BSI defined according to the specific criteria of the National Healthcare Safety Network Dialysis Event Surveillance Manual (Centers for Disease Control and Prevention) [17], which defines BSI as any positive blood culture, were considered for enrollment in the study. We considered all positive blood cultures regardless of the site where blood was drawn. For common skin commensal pathogens (Staphylococcus coagulase negative including Staphylococcus epidermidis), we considered BSI when pathogens were cultured from two or more blood cultures and/or the patient was treated by a physician $[17,18]$. Blood cultures were processed in accordance with the recommendations of the Clinical and Laboratory Standards Institute [19].

The cases were obtained from a survey of microbiology laboratory records at the institution during the study period. For patients who had more than one positive blood culture, only the first episode was considered for this study. These patients were included in the case group. Hemodialysis patients at the same institution, $\geq 18$ years of age, and without positive blood cultures during the study period were used as controls. Pairing criteria were: age, time on hemodialysis, and diagnosis of diabetes mellitus. The proportion of cases and controls was 1:1.

\section{Study protocol and data collection}

Data collection occurred through review of patients' medical records. For the cases group, data collection included sociodemographic characteristics, comorbidities, body mass index [20], microorganisms in blood culture, resistance profiles of antimicrobials, and previous treatment for end stage renal disease. The type, location and time of current and previous venous access, clinical complications (transfusions, number of transfusions, hospitalization because of infection, length of hospital stay, prior antimicrobial use, previous hospitalization, and number of previous hospitalizations), occurrence of death, and transfer of treatment were recorded for a period of 6 months prior to the BSI.

We defined short duration CVC as non-tunneled hemodialysis CVC. However some patients remained with this kind of catheter for a longer period due to difficult inserting other more adequate vascular access for hemodialysis. Long duration $\mathrm{CVC}$ was defined as tunneled hemodialysis CVC [17]. The time of current and previous venous accesses was stratified by period. This was done as in some situations they may present with similar periods.

Prior antimicrobial use was defined as every previous antimicrobial treatment for at least seven days.

Death was considered to be related to BSI when it occurred within 15 days after microbiologic diagnosis of infection [21,22]. 
Controls were selected from the computerized patient record system, according to the previously mentioned pairings, using the closest date of beginning dialysis in relation to the case group. Of these, the same variables described above were collected, except for the presence of BSI at the beginning of the study.

A microorganism was considered multi-resistant when it showed resistance to a pharmaceutical agent in three or more classes of antimicrobials. Staphylococcus was considered multi-resistant when it showed no susceptibility to methicillin, and Enterococcus was considered multi-resistant when it showed no susceptibility to vancomycin (VRE) [23-25].

\section{Statistical analysis}

A descriptive analysis of the cases and controls was conducted which examined the variables of interest. Data are presented using absolute frequencies and percentages for categorical variables, and mean \pm standard deviation for continuous variables. The primary outcome was BSI. Associations between BSI and categorical variables were tested using the chi-square test, Fisher's exact test or likelihood ratio. The association between the continuous variables and the presence of BSI was examined using the student's $t$-test or Mann-Whitney test, as appropriate. Univariate logistical regression was used to investigate the relationship between each independent variable (preselected at the crossings where $\mathrm{p} \leq 0.05$ ) and the dependent variable, BSI. To compare the outcomes of patients that developed BSI and the patients that did not developed BSI we used only the univariate logistical regression.

Multiple logistic regression analysis was conducted to determine the independent variables that continued to be associated with BSI after inclusion in the model of significant variables in univariate analysis. To include the variables in the regression model, the forward stepwise method was used. Odds ratios were calculated with respective confidence intervals of $95 \%$. SPSS version 19.0 (Chicago, IL, USA) was used for statistical analyses.

\section{Results}

The total number of patients in the dialysis program for the study period was 353, of which 221 (62\%) were on hemodialysis and $132(38 \%)$ were on peritoneal dialysis. Ninety-three patients (42.1\%) met inclusion criteria. Twelve patients were excluded because they were unable to be paired with a control. The final study population included 162 patients (81 cases and 81 controls). For nine cases, a control of the same age was not available, and for three cases, a control with the same time on hemodialysis was not available.

The sociodemographic characteristics of patients included in the study are presented in Table 1. There was
Table 1 Sociodemographic characteristics of the cases group and control patients

\begin{tabular}{llll}
\hline & Case $(\boldsymbol{n}=\mathbf{8 1})$ & Control $(\boldsymbol{n}=\mathbf{8 1})$ & $\boldsymbol{p}$-value \\
\hline Age & $56 \pm 17.1$ & $53 \pm 16.3$ & 0.325 \\
Male & & & \\
Female & $36(44.5)$ & $47(58.0)$ & 0.083 \\
Race & $45(55.5)$ & $34(42.0)$ & \\
White & & & \\
Nonwhite & $49(60.5)$ & $55(68.0)$ & 0.325 \\
Educational level & $32(39.5)$ & $26(32.0)$ & \\
No education & $21(26.0)$ & $14(17.0)$ & 0.198 \\
Elementary & $28(34.5)$ & $22(27.0)$ & \\
High School & $22(27.0)$ & $28(35.0)$ & \\
Higher education & $10(12.5)$ & $17(21.0)$ & \\
\hline
\end{tabular}

Values are expressed as mean \pm SD or $n(\%)$.

no statistically significant difference in these characteristics among the study population.

For clinical data related to dialysis treatment, the following variables were statistically significant $(p \leq 0.05)$ in the initial analyses and were preselected for logistic regression: associated diseases (hypertension), previous treatment (history of peritoneal dialysis), current type of venous access, duration of current venous access, use of previous venous access, previous type and duration of tunneled hemodialysis CVC, previous use of antimicrobials, and previous hospitalization. Duration of previous venous access had a $\mathrm{p}$-value of $\leq 0.05$; however, it was not included in regression analysis because of its low frequency (Table 2).

Table 3 shows the results of logistic regression analysis. In univariate analysis, variables significantly associated with the occurrence of BSI were: hypertension, history of peritoneal dialysis, type and duration of current venous access, use of a tunneled hemodialysis $\mathrm{CVC}$ as previous venous access, previous use of antimicrobials, and previous hospitalization (whether there had been a previous hospitalization or not).

With multiple logistic regression analysis, current access (CVC) and previous hospitalization were independent risk factors for the occurrence of BSI. Patients who had a CVC showed an 11.2-fold (CI 95\%: 5.17-24.29) greater chance of developing BSI compared with patients who had an AVF. Previous hospitalization increased the chance of developing a BSI 6.6-fold (CI 95\%: 1.9-23.09).

The most prevalent microorganisms isolated in blood cultures taken from the case group were Gram-positive bacteria (72.8\%), followed by Gram-negative bacteria (25.9\%), and fungi (1.2\%). Among the Gram-positive microorganisms, S. aureus was isolated in $32.1 \%$ of cases, and of these, $38.5 \%$ showed resistance to methicillin. 
Table 2 Clinical variables of interest of the case group and control patients

\begin{tabular}{|c|c|c|c|}
\hline & Case $(n=81)$ & Control $(n=81)$ & $p$-value \\
\hline \multicolumn{4}{|l|}{ Comorbidities } \\
\hline Hypertension & $71(87.6)$ & $59(72.8)$ & 0.018 \\
\hline Cardiovascular diseases & $17(20.9)$ & $12(14.8)$ & 0.306 \\
\hline Diabetes mellitus & $26(32.0)$ & 26(32.0) & 1.000 \\
\hline Others* & $27(33.3)$ & $22(27.2)$ & 0.421 \\
\hline Body mass index, $\mathrm{Kg} / \mathrm{m}^{2}$ & $24 \pm 4.3$ & $24 \pm 3.9$ & 0.945 \\
\hline Previous treatments & $56(69.1)$ & $47(58.0)$ & 0.141 \\
\hline \multicolumn{4}{|l|}{ Type of previous treatments } \\
\hline Conservative & $36(64.3)$ & $30(37.0)$ & 0.337 \\
\hline Peritoneal dialysis & $19(33.9)$ & $9(11.1)$ & 0.038 \\
\hline Hemodialysis & $17(30.4)$ & $9(11.1)$ & 0.087 \\
\hline Transplant & $15(26.8)$ & $8(9,9)$ & 0.115 \\
\hline Duration of hemodialysis treatment, months & $29 \pm 39.2$ & $36 \pm 42.1$ & 0.276 \\
\hline \multicolumn{4}{|l|}{ Type of current venous access } \\
\hline$A V F^{* *}$ & $25(30.9)$ & $64(79.0)$ & $<0.001$ \\
\hline Tunneled hemodialysis CVC ${ }^{* * *}$ & $52(64.2)$ & $13(16.0)$ & \\
\hline Non-tunneled hemodialysis CVC & $4(4.9)$ & $1(1.2)$ & \\
\hline PTFE Graft ${ }^{* * * *}$ & $0(-)$ & $3(3.7)$ & \\
\hline \multicolumn{4}{|l|}{ Location of current venous access } \\
\hline Upper arm & $24(29.6)$ & $67(82.7)$ & \\
\hline Jugular vein & $50(61.7)$ & $13(16.0)$ & \\
\hline Subclavian vein & $7(8.6)$ & $0(-)$ & \\
\hline Femoral vein & $0(-)$ & $1(1.2)$ & \\
\hline \multicolumn{4}{|l|}{ Duration of current venous access } \\
\hline $0-30$ & $17(21,0)$ & $3(3.7)$ & $<0.001$ \\
\hline 30-180 days & $27(33.3)$ & $8(9.9)$ & \\
\hline$>180$ days & $37(45.7)$ & $70(86.4)$ & \\
\hline Previous venous access ( 6 months) & $26(32.1)$ & $11(13.6)$ & 0.005 \\
\hline Number of previous accesses & $1.2 \pm 0.41$ & $1.3 \pm 0.46$ & 0.744 \\
\hline Type of previous venous access & & & \\
\hline AVF & $7(8.6)$ & $3(3.7)$ & 0.192 \\
\hline Tunneled hemodialysis CVC & $14(17.3)$ & $5(6.2)$ & 0.028 \\
\hline Non-tunneled hemodialysis CVC & $5(6.2)$ & $6(7.4)$ & 0.755 \\
\hline PTEF Graft & $5(6.2)$ & $1(1.2)$ & 0.210 \\
\hline Location of tunne & & & \\
\hline Jugular vein & $13(16.0)$ & $3(3.7)$ & \\
\hline Subclavian vein & $0(-)$ & $1(1.2)$ & \\
\hline Location of non-t & & & \\
\hline Jugular vein & $4(4.9)$ & $6(7.4)$ & \\
\hline Subclavian vein & $1(1.2)$ & 0 & \\
\hline
\end{tabular}


Table 2 Clinical variables of interest of the case group and control patients (Continued)

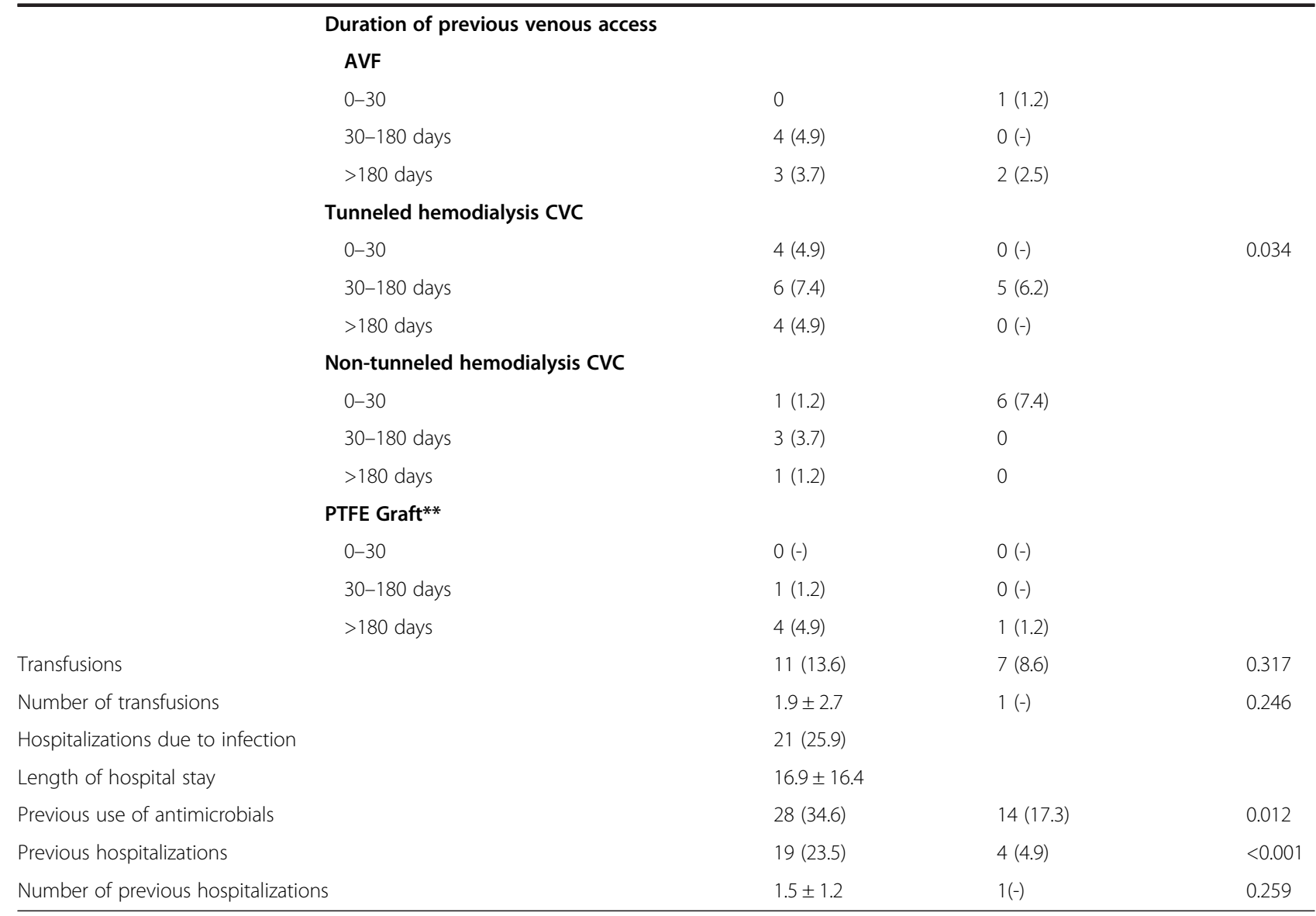

Values are expressed as mean \pm SD or $n(\%) .{ }^{*}$ hypothyroidism, hyperthyroidism, neoplasm and systemic lupus erythematosus; **AVF: arteriovenous fistula;

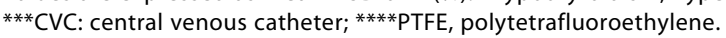

Table 3 Univariate and multivariate logistic regression analyses

\begin{tabular}{|c|c|c|c|c|}
\hline & \multicolumn{2}{|c|}{ Simple logistic regression } & \multicolumn{2}{|c|}{ Multiple logistic regression } \\
\hline & OR (IC 95\%) & $p$-value & OR (IC 95\%) & $p$-value \\
\hline Diagnosis of hypertension & $2.65(1.16-6.03)$ & 0.021 & & \\
\hline Previous treatment (peritoneal dialysis) & $2.45(1.03-5.81)$ & 0.042 & & \\
\hline \multicolumn{5}{|l|}{ Type of current venous access } \\
\hline $\mathrm{CVC}^{*}$ Vs. AVF** & $10.72(5.09-22.57)$ & $<0.001$ & $11.2(5.17-24.29)$ & $<0.001$ \\
\hline \multicolumn{5}{|l|}{ Duration of current venous access } \\
\hline $0-30$ vs. $>180$ days & $10.72(2.95-38.96)$ & $<0.001$ & & \\
\hline 30-180 days vs. $>180$ days & $6.39(2.64-15.45)$ & $<0.001$ & & \\
\hline Use of previous venous access & $3.01(1.37-6.62)$ & 0.006 & & \\
\hline Tunneled CVC (previous access) & $3.18(1.09-9.28)$ & 0.035 & & \\
\hline Prior antimicrobial use & $2.53(1.21-5.28)$ & 0.013 & & \\
\hline Previous hospitalizations & $5.90(1.91-18.24)$ & 0.002 & $6.63(1.9-23.09)$ & 0.003 \\
\hline
\end{tabular}

*CVC: central venous catheter; ${ }^{* *}$ AVF: arteriovenous fistula. 
The second most frequent Gram-positive organism was S. epidermidis (13.6\%), with $100 \%$ resistance to methicillin. Of the Gram-negative microorganisms isolated, $13.6 \%$ were of the enterobacteriaceae family and $12.3 \%$ were non-fermenters. For Gram-negative microorganisms, 33.3\% were multi-resistant (Table 4).

MRSA was isolated in 10 patients. Two of these patients had previous exposure to vancomycin and had a minimum inhibitory concentration to vancomycin of $\leq 0.05 \mu \mathrm{g} / \mathrm{mL}$ and $1.0 \mu \mathrm{g} / \mathrm{mL}$.

With regard to progression for cases and controls, there were no significant differences in variables. Death occurred only in the cases group, retention in treatment was higher in controls, and shift to peritoneal dialysis showed a higher frequency in the cases group. Fifteen patients $(46.7 \%)$ died from BSI, two (13.3\%) from cardiovascular disease, and six (40\%) from other causes, such as neoplasms, infections at other sites, and noninfectious complications at the vascular access. The median time to death after BSI was $4.6 \pm 5.5$ days (Table 5). Among the patients that died due to other causes, the death occurred from 45 to 672 days after the BSI.

\section{Discussion}

For patients undergoing renal replacement therapy, infection is the leading cause of morbidity and the second leading cause of mortality [1]. In Brazil, it is estimated that 97,586 patients currently receive chronic dialysis treatment [26]. According to North American data, the

Table 4 Microorganisms isolated in the cases group, and profiles of resistance

\begin{tabular}{llll}
\hline & MR & S & Total \\
\hline Gram-positives & $28(47.5)$ & $31(52.5)$ & $59(72.8)$ \\
Staphylococcus aureus & $10(38.5)$ & $16(61.5)$ & $26(32.1)$ \\
Staphylococcus epidermidis & $11(100.0)$ & $0(-)$ & $11(13.6)$ \\
Staphylococcus coagulase & $4(57.1)$ & $3(42.9)$ & $7(8.6)$ \\
negative non S. epidermidis & & & $3(3.7)$ \\
Enterococcus faecalis & $1(33.3)$ & $2(66.7)$ & $12(14.8)$ \\
Other Gram-positives* & $3(33.3)$ & $9(66.7)$ & $21(25.9)$ \\
Gram-negatives & $7(33.3)$ & $14(66.7)$ & $11(13.6)$ \\
Enterobacteriaceae** & $4(36.4)$ & $7(63.6)$ & $10(12.3)$ \\
Non-fermenters*** & $3(30.0)$ & $7(70.0)$ & $1(1.2)$ \\
Fungi & $0(-)$ & $1(100.0)$ & $1(1.2)$ \\
Candida albicans & $0(-)$ & $1(100.0)$ & 81 \\
Total of microorganisms & & &
\end{tabular}

Values are expressed as mean \pm SD or $n$ (\%). MR: multi-resistant. S: sensitive. *Streptococcus acidominimus, Streptococcus agalactiae, Streptococcus anginosus, Streptococcus bovis, Streptococcus pyogenes, Staphylococcus simulans, Staphylococcus capitis, Staphylococcus haemolyticus, Staphylococcus lugdunensis; **Enterobacteriaceae: Enterobacter spp, Enterobacter aerogenes, Enterobacter cloacae, Escherichia coli, Klebsiella pneumoniae, Serratia marcescens, Proteus mirabilis; ${ }^{* * *}$ Non-fermenters: Acinetobacter baumannii, Pseudomonas aeruginosa, Stenotrophomonas maltophilia.
Table 5 Progression of the patients, cause of death and time of death post-BSI

\begin{tabular}{|c|c|c|c|}
\hline & $\begin{array}{l}\text { Cases } \\
(n=81)\end{array}$ & $\begin{array}{l}\text { Controls } \\
(n=81)\end{array}$ & $p$-value \\
\hline \multicolumn{4}{|c|}{ Progression of the patients } \\
\hline Death & $15(18.5)$ & $0(-)$ & $<0.001$ \\
\hline Transfer of care & $9(11.1)$ & $5(6.2)$ & 0.263 \\
\hline Duration of treatment & $41(50.6)$ & $70(86.4)$ & $<0.001$ \\
\hline Peritoneal dialysis & $9(11.1)$ & $1(1.2)$ & 0.009 \\
\hline Transplant & $7(8.6)$ & $5(6.2)$ & 0.549 \\
\hline \multicolumn{4}{|c|}{ Cause of death in the cases } \\
\hline BSI & $7(46.7)$ & & \\
\hline Cardiovascular diseases & $2(13.3)$ & & \\
\hline Other* & $6(40.0)$ & & \\
\hline Total & 15 & & \\
\hline
\end{tabular}

Time of death post-BSI

$4.6 \pm 5.5$

Values are expressed as mean \pm SD or $n$ (\%). *neoplasms, infections at other sites, cardiovascular diseases, and noninfectious complications in the vascular access.

rates of BSI among hemodialysis patients range between 0.5 and 27.1 per 100 patients/month depending on the type of venous access used [2]. Results of a Brazilian study reported that rates varied between 0.55 and 7.32 per 1000 venous accesses/day [27].

In the present study, risk factors for BSI were evaluated in patients on hemodialysis at a hemodialysis service in São Paulo, Brazil. The study was a case-control design, which can analyze risk factors related to a determined condition. Patients presenting with BSI during the study period were included as cases, and controls were those patients who did not develop the studied event (pairing criteria: age, previous time on hemodialysis, and diagnosis of diabetes mellitus).

Use of a CVC compared with use of an AVF, and previous hospitalization were independent risk factors for the occurrence of BSI among patients on hemodialysis treatment. Use of a CVC was associated with an increased risk of developing BSI (OR: 11.2; CI 95\%: 5.17-24.29; p < 0.001) compared with the use of an AVF. This finding aligns with previous trials of antimicrobial locks, which reported a higher occurrence of BSI among hemodialysis patients with CVC (3-4 cases per 1000 catheter-days) [28]. In a longitudinal cohort study, Xui et al. found that the rates of BSI in patients using CVC were three times higher than in patients using AVF $(\mathrm{p}<0.001)$ [8]. A case-control study conducted at a university hospital in Greece demonstrated, through multivariate logistic regression, that patients using CVC had a higher risk of BSI compared with patients using AVF (OR: 2.93; $\mathrm{p}=0.047$ ) [10].

In the current study, only five patients (four cases and one control) used a non-tunneled hemodialysis CVC. In 
these patients, three had started hemodialysis within 60 days and were awaiting the manufacture of an AVF, and the other two had been undergoing treatment for a longer period of time and had spent longer with a nontunneled hemodialysis CVC because of difficulties in the insertion of a tunneled hemodialysis CVC or the manufacture of an AVF.

Previous hospitalization increased the risk of BSI 5.33fold $(p=0.003)$. There are few reports in the literature on the association between BSI and previous hospitalizations in patients undergoing hemodialysis treatment. Nguyen et al. in a study performed between 2005 and 2011, evaluated BSI from MRSA in patients on dialysis treatment, and found that $70 \%$ of patients who developed BSI were hospitalized at least once in the year prior to the infection [6]. In a study by Barbosa et al. the researchers reported that, in 320 chronic renal patients, previous hospitalization was an important risk factor for colonization of multi-resistant microorganisms (e.g., VRE) [29]. And Aktas et al. in a study of 70 patients on dialysis treatment, reported a higher colonization for MRSA in patients who had been hospitalized within the previous 6 months compared with those who had not [30].

In univariate logistic regression analysis, systemic arterial hypertension, peritoneal dialysis as a prior treatment, duration of venous access, presence of previous venous access, use of tunneled hemodialysis CVC as a previous access, and previous antimicrobials were associated with a higher occurrence of BSI. No data was found in the literature on the possible relationship between systemic arterial hypertension and previous peritoneal dialysis with the occurrence of BSI in this population.

For examination of time of duration of current venous access as a risk factor, BSI was found to be associated with access that had been gained within 30 days. Duration of current venous access was analyzed using three categories ( $0-30$ days, $30-180$ days, and $>180$ days). BSI was higher in those who had had the CVC inserted within 30 days, which underscores the need for greater care during the CVC insertion procedure. Napalkov et $a l$. in a study that evaluated infectious and noninfectious complications in CVC, reported that the majority of BSI occurred within the first 90 days, with an incidence rate/ 1000 catheter-days of 5.1 (CI 95\%: 3.7-4.3) [31]. There was also a higher risk of infection for catheters inserted within the 6 months following infection, reinforcing the need for care during this period.

The use of previous venous access and, primarily, previous access of tunneled hemodialysis CVC, was associated with a higher occurrence of BSI. As mentioned above, studies have reported that CVC use is associated with a higher incidence of BSI compared with the use of an AVF [8-10]. References to previous venous access as a risk factor for BSI were not found in the literature.
Previous use of antimicrobials was associated with a higher occurrence of BSI (OR: 2.53; $\mathrm{p}=0.013$ ). As with other variables, data were collected on the use of antimicrobials within 6 months before the event. We found no specific studies that related prior antimicrobial exposure to BSI in patients undergoing hemodialysis treatment. However, Lim et al. in a case-control study conducted with patients seen in the emergency department of a tertiary hospital in Australia, showed through multivariate analysis that prior antimicrobial use increased the risk of developing BSI by multi-resistant microorganisms (OR: 5.49; $\mathrm{p}<0.001$ ) [32]. According to the Centers for Disease Control and Prevention, the rational use of antimicrobials is an important measure for controlling the spread of multi-resistant microorganisms [23].

Gram-positive microorganisms were the most prevalent (72.8\%) found in the current study. Among them, S. aureus was the most frequently isolated (32.1\%), with about $38.5 \%$ resistant to oxacillin. Other studies have also reported a high prevalence of Gram-positive microorganisms in patients undergoing hemodialysis treatment, primarily S. aureus $[7,10,11]$. Despite the high prevalence of Grampositive microorganisms observed in the current study, Gram-negative microorganisms accounted for $25.9 \%$ of isolates in blood cultures. In a university hospital in Spain, microorganisms isolated in blood cultures from patients with kidney disease were: Gram-negative bacteria, 52.3\%; Gram-positive bacteria, 46.5\%; and fungi, 1.2\%. Escherichia coli was the most frequent microorganism (27\%) [33].

With regard to resistance profiles, microorganisms such as MRSA and VRE have been isolated in surveillance cultures collected from patients on dialysis treatment $[29,34,35]$. Aktas et al. used molecular typing to show a high similarity between MRSA strains isolated from surveillance cultures and clinical cultures collected from patients on dialysis treatment [30]. And a study conducted at long-term care facilities in Hong Kong reported a significant dissemination of MRSA, reinforcing the need to adopt measures to reduce the transmission of this microorganism [36].

The independent risk factors for BSI identified in the current study were use of a CVC compared with use of an AVF, and previous hospitalization. Although previous studies have indicated a relationship between the presence of CVC and BSI, our study expands the characteristics of this group, highlighting the importance of care during the initial period and the period of up to 6 months of catheter duration. Furthermore, the study highlights the importance of previous hospitalization as a risk factor for BSI. To the best of the authors' knowledge, this factor has not been reported in previous studies and requires further research.

This study had some limitations inherent in the retrospective character of the data collection. However, patient 
records at the study institution were completed with significant caution, given the characteristics of a service dedicated to the treatment of chronic renal patients, and followed the high standards of the organization. Additionally, as there are standardized protocols for performing blood cultures for these patients, it is unlikely that events were underdiagnosed in the study period.

Preventative measures against BSI related to CVC should be strengthened and effectively applied to hemodialysis treatment units, as well as reducing the use of this device whenever possible, prioritizing the use of AVF. Considering that previous hospitalization was an independent risk factor for BSI, patients who developed BSI after hospitalization are probably infected by microorganisms during this period. Standard precautions should be enforced to prevent microorganism dissemination during hospitalization.

A reduction in the number of infections in this population may contribute to a decrease in hospital admissions, since infections are a leading cause of hospitalization in patients on hemodialysis treatment.

\section{Conclusions}

The risk factors for BSI in chronic renal disease patients receiving hemodialysis were previous hospitalization and use of a CVC. The most prevalent microorganisms were Gram-positive bacteria, of which $47.5 \%$ were multi-resistant. The results of this study suggest the need to intensify measures to prevent and control BSI in hemodialysis patients, among which are specific measures for insertion and maintenance of the CVC, and the judicious use of this device. Efforts should be made to reduce exposure of these patients to hospitalization to minimize the risk of BSI. Future research could evaluate the impact of surveillance cultures to prevent microorganism dissemination in this population.

\section{Consent}

Written informed consent was obtained from the patient for the publication of this report and any accompanying images.

\section{Competing interests}

The authors declare that they have no competing interests.

\section{Authors' contributions}

DF: principal researcher and participated in all steps of the study: development of the project, data collection, revision of the database, data analysis, and drafting and revising the manuscript. MFPO: development of the project, data analysis and article revision. MT: development of the project, data analysis and article revision. VP: development of the project, data analysis, and article revision. SRM: data collection and revision of the database. CG: revision of the database and article revision. AB: data analysis and article revision. RS: manuscript structure and article revision. DB: coordinated and conceived the study, participated in project design, supervision of data collection, data analysis, drafting and revision of the article. All authors read and approved the final version of the manuscript.

\section{Authors' information}

DF: Nurse, MSc and PhD; MFPO: Nurse, MSc and PhD; MT: Nurse, MSc, PhD and post- doctoral student (EPE/UNIFESP); VP: Physician, MSc and doctoral student (EPM/UNIFESP); SRM: Nurse, MSC and doctoral student (EPE/UNIFESP); CG: Nurse, PhD and post-doctoral student (EPE/UNIFESP); AB: Nurse, MSc, PhD and Adjunct Professor (EPE/UNIFESP); RS: Physician, MD, PhD and Associate Professor (EPM/UNIFESP); and, DB: Nurse, PhD, MSC and Associate Professor (EPE/UNIFESP).

\section{Acknowledgements}

We wish to acknowledge the Fundação de Amparo à Pesquisa do Estado de São Paulo FAPESP and Conselho Nacional de Desenvolvimento Científico e Tecnológico CNPq for the financial support for this study.

\section{Author details}

${ }^{1}$ School of Nursing, Paulista School of Nursing, Universidade Federal de São Paulo (Federal University of São Paulo - EPE/UNIFESP), R. Napoleão de Barros 754, São Paulo 04024-002, Brazil. 'Infection Control Unit, Children's Institute and Institute for the Treatment of Childhood Cancer, School of Medicine, Universidade de São Paulo (University of São Paulo - ITACI/FMUSP), Av. Dr. Enéas Carvalho de Aguiar 647, São Paulo 05403-000, Brazil. ${ }^{3}$ Division of Infectious Diseases, Escola Paulista de Medicina, Universidade Federal de São Paulo (Paulista School of Medicine, Federal University of São Paulo - EPM/ UNIFESP), R. Napoleão de Barros, 715, $7^{\circ}$ andar, São Paulo 04024-002, Brazil. ${ }^{4}$ Division of Dialysis, Hospital do Rim e Hipertensão, Fundação Oswaldo Ramos, (Kidney and Hypertension Hospital, Foundation Oswaldo Ramos HRIM/FOR), R. Pedro de Toledo 282, São Paulo 04039-030, Brazil. ${ }^{5}$ Division of Nephrology, Paulista School of Medicine, Universidade Federal de São Paulo (Federal University of São Paulo - EPM/UNIFESP), R. Botucatu 740, São Paulo 04023-900, Brazil.

Received: 23 December 2014 Accepted: 13 March 2015

Published online: 26 March 2015

\section{References}

1. United States Renal Data System. U.S. Renal Data System: USRDS 2013 Annual Data Report: Atlas of Chronic Kidney Disease and End-Stage Renal Disease in the United States, National Institutes of Health, National Institute of Diabetes and Digestive and Kidney Diseases. Bethesda, MD; 2013. [http://www.usrds.org/atlas.aspx]

2. Klevens RM, Edwards JR, Andrus ML, Peterson KD, Dudeck MA, Horan TC et al. Dialysis Surveillance Report: National Healthcare Safety Network (NHSN)-data summary for 2006. Semin Dial. 2008;21 Suppl 1:24-8.

3. Centers for Diseases Control and Prevention. Vital signs: central line-associated blood stream infections- United States, 2001, 2008, and 2009. MMWR. 2011;60:243-8

4. Patel PR, Yi SH, Booth S, Bren V, Downham G, Hess S, et al. Bloodstream infection rates in outpatient hemodialysis facilities participating in a collaborative prevention effort: a quality improvement report. Am J Kidney Dis. 2013;62 Suppl 2:322-30.

5. National action plan to reduce healthcare-associated infections [http:// www.hhs.gov/ash/initiatives/hai/actionplan/index.html]

6. Nguyen DB, Lessa FC, Belflower R, Mu Y, Wise M, Nadle J, et al. Invasive methicillin-resistant Staphylococcus aureus infections among patients on chronic dialysis in the United States, 2005-2011. Clin Infect Dis. 2013;57 Suppl 10:1393-400.

7. Gauna TT, Oshiro E, Luzio YC, Paniago AM, Pontes ER, Chang MR. Bloodstream infection in patients with end-stage renal disease in a teaching hospital in central-western Brazil. Rev Soc Bras Med Trop. 2013;46 Suppl 4:426-32.

8. Xue H, Ix JH, Wang W, Brunelli SM, Lazarus M, Hakim R, et al. Hemodialysis access usage patterns in the incident dialysis year and associated catheter-related complications. Am J Kidney Dis. 2013;61 Suppl 1:123-30.

9. Patel PR, Kallen AJ, Arduino MJ. Epidemiology, surveillance, and prevention of bloodstream infections in hemodialysis patients. Am J Kidney Dis. 2010;56 Suppl 3:566-77.

10. Fysaraki M, Samonis G, Valachis A, Daphnis E, Karageorgopoulos DE, Falagas $M E$, et al. Incidence, clinical, microbiological features and outcome of bloodstream infections in patients undergoing hemodialysis. Int J Med Sci. 2013;10 Suppl 12:1632-8.

11. Esmanhoto CG, Taminato M, Fram DS, Belasco AGS, Barbosa DA. Microorganisms isolated from patients on hemodialysis by central venous 
catheter and related clinical evolution. Acta Paul Enferm. 2013;26 Suppl 5:413-20.

12. Fram D, Taminato M, Ponzio V, Manfredi SR, Grothe C, Batista RE, et al. Risk factors for morbidity and mortality of bloodstream infection in patients undergoing hemodialysis: a nested case-control study. BMC Res Notes. 2014;7(Supp 1):882

13. Fram DS, Taminato M, Ferreira D, Neves L, Belasco AGS, Barbosa DA. Prevention of Catheter-Related Bloodstream Infections in Patients on Hemodialysis. Acta Paul Enferm. 2009;22:564-8.

14. Pugh-Clarke K, Donlon S, McCann M. CE: continuing education article. Prevention of infection in patients with chronic kidney disease part 1: application of infection control principles to the renal care environment. J Ren Care. 2010;36 Suppl 4:191-8.

15. Centers for Disease Control and Prevention. Infection control requirements for dialysis facilities and clarification regarding guidance on parenteral medication vials. MMWR. 2008:57 Suppl 32:875-6.

16. Centers for Disease Control and Prevention. Recommendations for preventing transmission of infections among chronic hemodialysis patients. MMWR. 2001;50:1-43.

17. Centers for Disease Control and Prevention. Dialysis Event Surveillance Manual. Atlanta; 2013. [http://www.cdc.gov/nhsn/PDFs/pscManual/DialysisManual.pdf]

18. Horan TC, Andrus M, Dudeck MA. CDC/NHSN surveillance definition of health care-associated infection and criteria for specific types of infections in the acute care setting. Am J Infect Control. 2008;36 Suppl 5:309-32.

19. Clinical and Laboratory Standards Institute. Performance Standards for Antimicrobial Susceptibility Testing; 23st Informational Supplement. CLSI document M100-S23. Wayne, PA: Clinical and Laboratory Standards Institute; 2013

20. de Onis M, Habicht JP. Anthropometric reference data for international use: recommendations from a World Health Organization Expert Committee. Am J Clin Nutr. 1996;64(4):650-8.

21. Conterno LO, Wey SB, Castelo A. Risk factors for mortality in Staphylococcus aureus bacteremia. Infect Control Hosp Epidemiol. 1998;19 Suppl 1:32-7.

22. Lee $\mathrm{CH}$, Chien CC, Liu JW. Timing of initiating glycopeptide therapy for methicillin-Resistant Staphylococcus aureus bacteremia: the impact on clinical outcome. Sci World J. 2013;2013:457435.

23. Center for disease control and prevention. Antibiotic resistance threats in the United States. Atlanta; 2013. [http://www.cdc.gov/drugresistance/threatreport-2013/pdf/ar-threats-2013-508.pdf]

24. Kallen AJ. Identifying and classifying bloodstream infections among hemodialysis patients. Semin Dial. 2013;26 Suppl 4:407-15.

25. Center for disease control and prevention: Guideline for Isolation Precautions. Preventing Transmission of Infectious Agents in Healthcare Settings. Atlanta; 2007. [http://www.cdc.gov/hicpac/pdf/isolation/Isolation2007.pdf]

26. Sociedade Brasileria de Nefrologia. Censo de Diálise. São Paulo; 2012. [http://www.censo-sbn.org.br/inicio]

27. de Albuquerque SE, Cavalcante RD, Ponce D, Fortaleza CM. Epidemiology of healthcare-associated infections among patients from a hemodialysis unit in southeastern Brazil. Braz J Infect Dis. 2014;18 Suppl 3:327-30.

28. James MT, Conley J, Tonelli M, Manns BJ, MacRae J, Hemmelgarn BR, et al. Meta-analysis: antibiotics for prophylaxis against hemodialysis catheterrelated infections. Ann Intern Med. 2008;148 Suppl 8:596-605.

29. Barbosa D, Lima L, Silbert S, Sader H, Cendoroglo M, Draibe S, et al. Evaluation of the prevalence and risk factors for colonization by vancomycin-resistant Enterococcus among patients on dialysis. Am J Kidney Dis. 2004;44 Suppl 2:337-43.

30. Aktaş E, Pazarli O, Külah C, Cömert F, Külah E, Sümbüloğlu V. Determination of Staphylococcus aureus carriage in hemodialysis and peritoneal dialysis patients and evaluation of the clonal relationship between carriage and clinical isolates. Am J Infect Control. 2011;39 Suppl 5:421-5.

31. Napalkov P, Felici DM, Chu LK, Jacobs JR, Begelman SM. Incidence of catheter-related complications in patients with central venous or hemodialysis catheters: a health care claims database analysis. BMC Cardiovasc Disord. 2013;13:86.

32. Lim CJ, Cheng AC, Kong DC, Peleg AY. Community-onset bloodstream infection with multidrug-resistant organisms: a matched case-control study. BMC Infect Dis. 2014;14 Suppl 1:126.

33. Rojas L, Muñoz P, Kestler M, Arroyo D, Guembe M, Rodríguez-Créixems M, et al. Bloodstream infections in patients with kidney disease: risk factors for poor outcome and mortality. J Hosp Infect. 2013;85 Suppl 3:196-205.
34. Fram D, Castrucci FM, Taminato M, Godoy-Martinez P, Freitas MC, Belasco A, et al. Cross-transmission of vancomycin-resistant Enterococcus in patients undergoing dialysis and kidney transplant. Braz J Med Biol Res. 2010;43 Suppl 1:115-9.

35. Gómez-González MC, Campos R, Pascua J, Marigliano N, Lancho JM, Teno P. Colonization management of methicillin resistant Staphylococcus aureus in patients and health professional in the haemodialysis unit in a situation of high risk of endemic disease: looking for zero nasal carriers? Enferm Infecc Microbiol Clin. 2012;30 Suppl 3:124-30.

36. Cheng VC, Tai JW, Wong ZS, Chen JH, Pan KB, Hai Y, et al. Transmission of methicillin-resistant Staphylococcus aureus in the long term care facilities in Hong Kong. BMC Infect Dis. 2013;13:205.

\section{Submit your next manuscript to BioMed Central and take full advantage of:}

- Convenient online submission

- Thorough peer review

- No space constraints or color figure charges

- Immediate publication on acceptance

- Inclusion in PubMed, CAS, Scopus and Google Scholar

- Research which is freely available for redistribution 